\title{
A novel IL-10-producing innate lymphoid cells (ILC10) in a contact hypersensitivity mouse model
}

\author{
Hyuk Soon Kim ${ }^{1, \#}$, Jong-Hwa Jang ${ }^{2, \#}$, Min Bum Lee ${ }^{1}$, In Duk Jung ${ }^{1}$, Yeong-Min Park ${ }^{1, *}$, Young Mi Kim ${ }^{3, *} \mathcal{E}^{2}$ Wahn Soo Choi $^{1, *}$ \\ ${ }^{1}$ Department of Immunology, School of medicine, Konkuk University, Chungju 27478, ${ }^{2}$ Department of Dental Hygiene, Hanseo University, \\ Seosan 31962, ${ }^{3}$ College of Pharmacy, Duksung Women's University, Seoul 01369, Korea
}

The immunoregulatory cytokine Interleukin 10 (IL-10) protein is produced by various cells during the course of inflammatory disorders. Mainly, it downregulates pro-inflammatory cytokines, antigen presentation, and helper $\mathrm{T}$ cell activation. In this study, we show that the ratio of IL-10-producing cells was significantly increased in lineage negative (i.e., not $\mathrm{T}, \mathrm{B}$, or leukocyte cell lineages) cells than in lineage positive cells in lymphoid and peripheral tissues. We further observed that IL-10-producing innate lymphoid cells (ILCs), here called firstly ILC10, were increased in number in oxazolone-induced contact hypersensitivity (CHS) mice. In detail, IL-10-producing lineage negative cells were elevated in the axillary, inguinal lymph node, and ear tissues of CHS mice. Notably, the cells expressed classical ILC marker proteins such as CD45, CD127, and Sca-1. Altogether, our findings suggest for the first time that ILC10s are present in various physiological settings and could be involved in numerous immune responses as regulatory cells. [BMB Reports 2016; 49(5): 293-296]

\section{INTRODUCTION}

Recent studies have shown that innate lymphoid cell (ILC) subsets participate in intestinal homeostasis, lymphoid organogenesis, cytotoxicity, tissue remodeling during wound healing, and early immune responses against foreign antigens by production of various cytokines or other mediators $(1,2)$. ILCs have been classified into three distinct subsets through

*Corresponding authors. Wahn Soo Choi, Tel: +82-2-2030-7813; Fax: +82-2-2049-6192; E-mail: wahnchoi@kku.ac.kr, Yeong-Min Park, Tel: +82-2-2049-6158; Fax: +82-2-2030-7845; E-mail: immun3023 @kku.ac.kr, Young Mi Kim, Tel: +82-2-901-8455; Fax: +82-2-9018164; E-mail: kym123@duksung.ac.kr

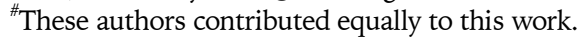

http://dx.doi.org/10.5483/BMBRep.2016.49.5.023

Received 3 February 2016, Revised 19 February 2016, Accepted 3 March 2016

Keywords: Contact hypersensitivity, ILC10, Innate lymphoid cells (ILCs), Interleukin-10, regulatory ILCs cytokine profiles and transcription factors. The groups are typically named ILCs (ILC1s), ILC2s, and ILC3s on the basis of their ability to produce $\mathrm{T}$ helper (Th) 1, Th2, and Th17 cell-linked cytokines, respectively $(3,4)$. Briefly, ILC1s express the transcription factor T-bet and produce interferon- $\gamma$ (IFN- $\gamma$ ) (5). ILC2s critically depend on transcription factor GATA3 for the development and production of Interleukin (IL)-5, IL-9, IL-13 and Amphiregulin (6-8). ILC3s require the orphan nuclear receptor RoRyt and produce IL-22 and IL-17A (9).

The cytokine IL-10 is well known for regulating effector $T$ cell immune responses, and inhibits the activation of myeloid lineage cells, such as suppression of MHC class II, B7-1/B7-2 expression, and pro-inflammatory cytokines production (10, 11). IL-10 is secreted by many different lymphoid and myeloid cells, such as T cells (particularly regulatory T cells), B cells (particularly regulatory B cells), natural killer (NK) cells, NKT cells, monocytes, macrophages, and dendritic cells (DCs) (12-17).

Although ILCs have been well recognized as the effector cells in various inflammatory immune responses, the subset of IL-10-producing ILCs (ILC10s) has not yet been reported. In this study, we demonstrated for the first time that the ILC10 subset is present in lymphoid tissues and is largely increased under disease conditions.

\section{RESULTS AND DISCUSSION}

In recent years, many studies on ILCs have focused on the characterization of their subsets through profiling of proinflammatory cytokine secretion and transcription factors $(2$, $3)$, but there was no evidence for a regulatory function of ILCs.

In general, it is well established that T cells, B cells, and other leukocytes act as effector cells to protect the host from foreign antigens, including various pathogens. Although the cells were initially identified as potent effector immune cells in the process of various immune diseases, other subsets were additionally recognized as regulatory cells $(10,12)$. In particular, some regulatory roles are dependent on IL-10 in Tr1, regulatory B10 cells, myeloid-derived suppressor cells, or type 2 macrophages (M2) (12-23). However, to the best of our knowledge, there is no previously published report on the presence of regulatory subsets of ILCs. 
We hypothesized that IL-10-producing ILC10s may exist in various immune tissues. In this study, we tested first whether or not IL-10-producing cells would exist in lineage negative cells, which excludes $\mathrm{T}$ cells $\left(\mathrm{CD} 3^{+}\right), \mathrm{B}$ cells $\left(\mathrm{CD} 45 \mathrm{R}^{+}\right)$, monocytes $\left(\mathrm{CD} 11 \mathrm{~b}^{+}\right)$, erythrocytes $\left(\mathrm{TER}-119^{+}\right)$, and neutrophils $\left(\mathrm{Gr}-1^{+}\right)$, in control or CHS mice. Interestingly enough, we observed that an $\mathrm{IL}-10^{+} \mathrm{Lin}^{-}$subset was definitely present, albeit rarely, in the spleen, axillary lymph node (aLN), inguinal lymph node (iLN), and ear (Fig. 1A). Furthermore, the ratio of IL-10 ${ }^{+} /$IL- $10^{-}$cells was significantly higher in Lin $^{-}$than in $\mathrm{Lin}^{+}$cells from second lymphoid tissues such as spleen, aLN, and iLN, but not ear tissue in control (ACE) mice. We also found that the ratio of $\mathrm{IL}-10^{+} / \mathrm{IL}-10^{-} \mathrm{Lin}^{-}$cells in aLN and ear tissues significantly increased in CHS mice (Figs. 1A and 1B). Accordingly, the frequency and number of $\mathrm{IL}-10^{+} \mathrm{Lin}^{-}$cells were substantially increased in the aLN, iLN, and ear, whereas the spleen was unaffected (Fig. 2), suggesting that mostly local drained LN IL-10 ${ }^{+} \mathrm{Lin}^{-}$cells, but not those from the spleen, are associated with inflammation in CHS mice.

A
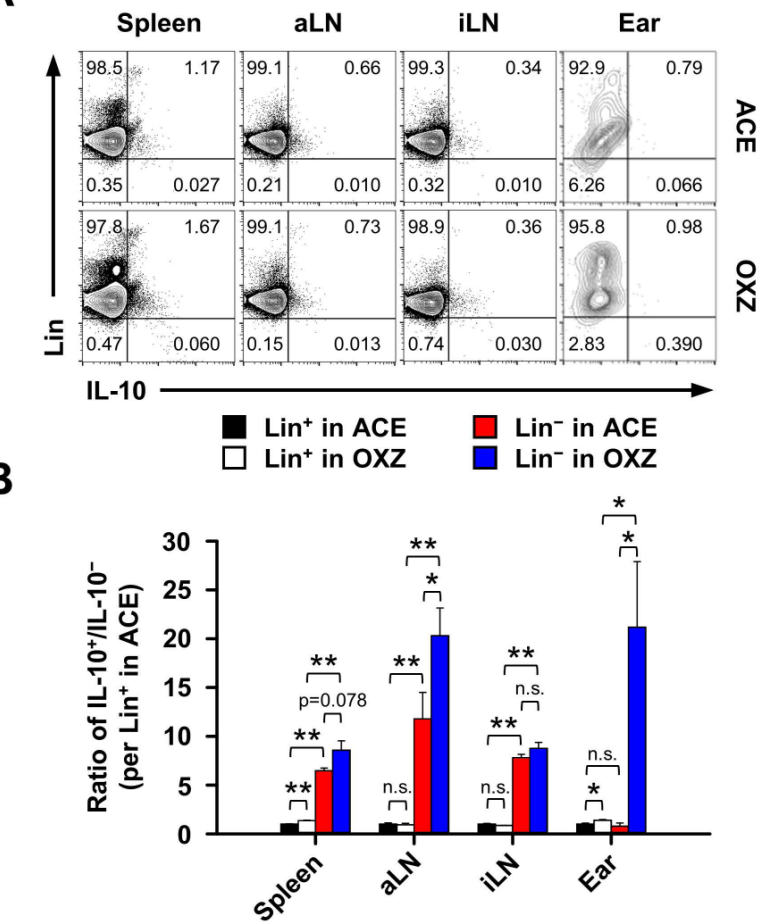

Fig. 1. IL-10 expression in lineage-negative cells in control or contact hypersensitivity mice. (A) Two days after being challenged with oxazolone, the frequency of lineage (Lin) ${ }^{+}$or $^{-}$and $\mathrm{IL}_{-10^{+}} \mathrm{or}^{-}$cells in spleen, aLN, iLN, and ear tissues was measured as described in "Materials and Methods." (B) The ratio of IL- $10^{+} / \mathrm{IL}-10^{-}$in $\mathrm{Lin}^{+}$or cells from contact hypersensitivity mice. Plots in (A) are representative images and data in $(B)$ are the mean \pm SEM from three independent experiments. ${ }^{*} \mathrm{P}<0.05$; $* * \mathrm{P}<0.01$; n.s., not significant. $\mathrm{OXZ}$, ozaxolone; ACE, acetone; aLN, axillary lymph node; iLN, inguinal lymph node.
The above results prompted us to investigate whether phenotypically $\mathrm{IL} 10^{+} \mathrm{Lin}^{-}$cells contain a new subset of ILCs (24). Notably, some subsets of $\mathrm{IL}_{-10} \mathrm{~L}^{+} \mathrm{Lin}^{-}$cells expressed several typical ILC markers such as CD45, CD127, and Sca-1, but CD25 was expressed in a low amount. We also observed that the expression of Sca- 1 was increased more in IL- $10^{+}$Lin $^{-}$ cells than in IL-10 ${ }^{-} \mathrm{Lin}^{-}$cells, but did not detect significant differences in CD45 and CD127 expression between IL-10 ${ }^{+}$ and IL-10 $\mathrm{Lin}^{-}$cells (Fig. 2A). We also observed no phenotypical difference in CD25 expression on the spleen, aLN, and iLN-derived IL- $10^{+}$or IL- $10^{-}$Lin $^{-}$cells. However, compared to IL-10 ${ }^{-}$Lin $^{-}$cells, the expression of CD25 was markedly increased in IL-10 ${ }^{+}$Lin $^{-}$cells in CHS-induced ear tissues (Fig. 3). Finally, we found that the number of IL-10 ILCs (ILC10, Lin $^{-} \mathrm{CD} 45^{+} \mathrm{CD} 127^{+} \mathrm{Sca}-1^{+}$) was significantly increased in the spleen, aLN, iLN, and ear in CHS mice (Fig. 4), suggesting that IL-10-producing ILC10s are associated with regulation of $\mathrm{CHS}$ inflammation. Given, particularly, the critical role of $\mathrm{T}$ cells in the progress of CHS inflammation (25), it would be of interest to determine whether ILC10s could regulate $T$ cells under pathological circumstances.

In summary, for the first time we identified the presence of a IL- $10^{+}$ILC $\left(\right.$Lin $\left.^{-} \mathrm{CD} 45^{+} \mathrm{CD} 127^{+} \mathrm{Sca}-1^{+}\right)$subset in lymphoid

A

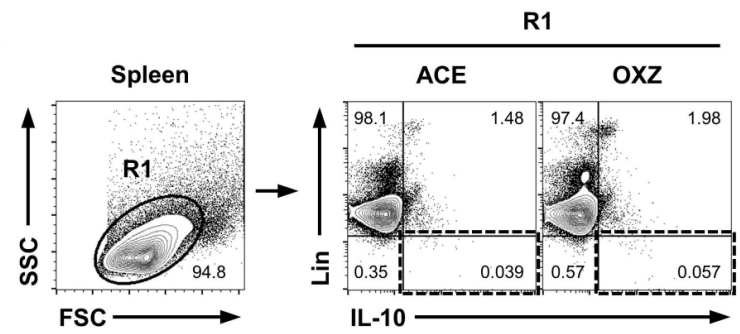

B

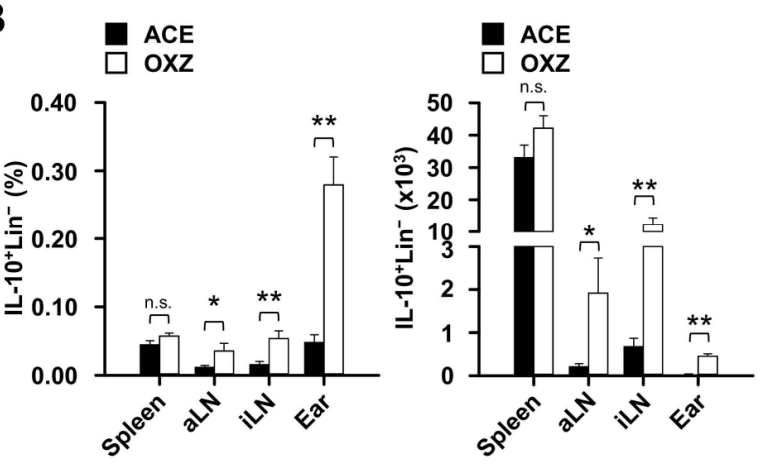

Fig. 2. IL-10 expression in lineage-negative cells from various tissues in the contact hypersensitivity mouse model. (A) Shown are the representative gating strategies for flow cytometry analysis of splenic IL-10 ${ }^{+}$Lin $^{-}$cells from contact hypersensitivity mice. (B) The frequency and count of $\mathrm{IL}_{-}-10^{+} \mathrm{Lin}^{-}$cells were determined by flow cytometric analysis as described in "Materials and Methods." Data are the mean \pm SEM from three independent experiments. ${ }^{*} \mathrm{P}<0.05 ;{ }^{*} \mathrm{P}<0.01$. n.s., not significant. OXZ, ozaxolone; ACE, acetone; aLN, axillary lymph node; iLN, inguinal lymph node. 


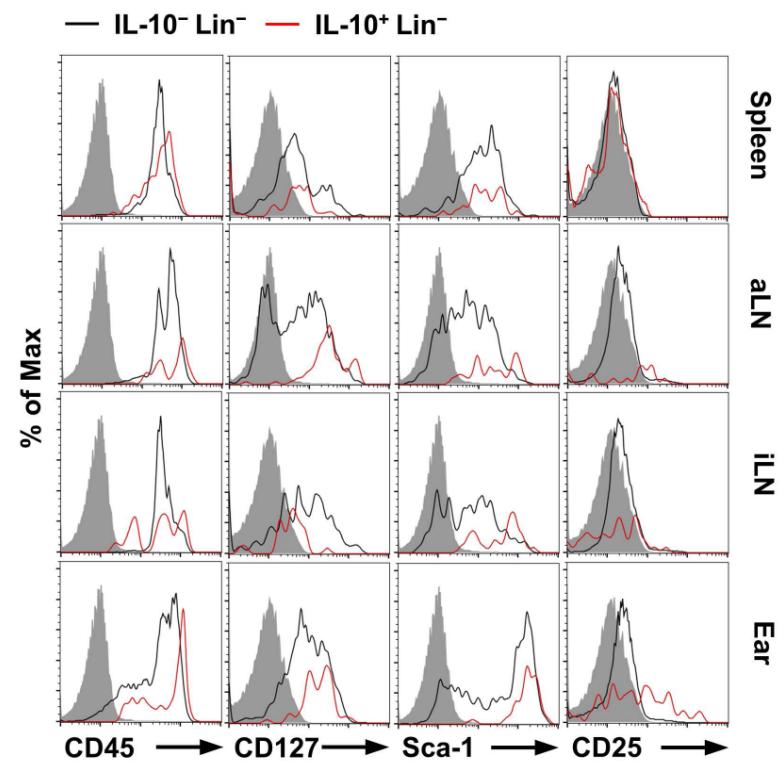

Fig. 3. Characterization of IL-10-producing lineage negative cells. Expressions of typical innate lymphoid cell surface markers on $\mathrm{IL}-10^{+}$ or IL-10 ${ }^{-}$in $\mathrm{Lin}^{-}$cells are shown. Histograms are representative images from three independent experiments. aLN, axillary lymph node; iLN, inguinal lymph node.

tissues, spleen, LN, and ear tissue, which we call ILC10. We further suggest that ILC10s could regulate some inflammatory immune responses in contact hypersensitivity and possibly other inflammatory diseases. Further studies are necessary to evaluate the physiology of ILC10s in various immune processes.

\section{MATERIALS AND METHODS}

\section{Mice}

C57BL/6 mice were purchased from Orientbio (Seongnam, Korea) and housed in a specific pathogen-free animal facility at Konkuk University (Seoul, Korea) with a sterilized diet and autoclaved water, unless indicated otherwise. The mice used were between 6 and 8 weeks of age. All animal experiments were approved by the Institutional Animal Care and Use Committee (IACUC) of Konkuk University.

\section{Induction of contact hypersensitivity in mice}

Contact Hypersensitivity $(\mathrm{CHS})$ was induced according to a previously reported method (18). Briefly, mice were sensitized with $25 \mu$ of Oxazolone $(100 \mathrm{mg} / \mathrm{ml}$, Sigma, St. Louis, MO) in Acetone/Olive Oil $(4: 1 \mathrm{v} / \mathrm{v})$ on the shaved hind flank for 2 consecutive days. Five days later, mice were challenged by the application of $10 \mu \mathrm{l}$ of oxazolone $(10 \mathrm{mg} / \mathrm{ml})$ on each ear. Two days after they were challenged with Oxazolone, single cells were isolated for flow cytometric analysis.
A
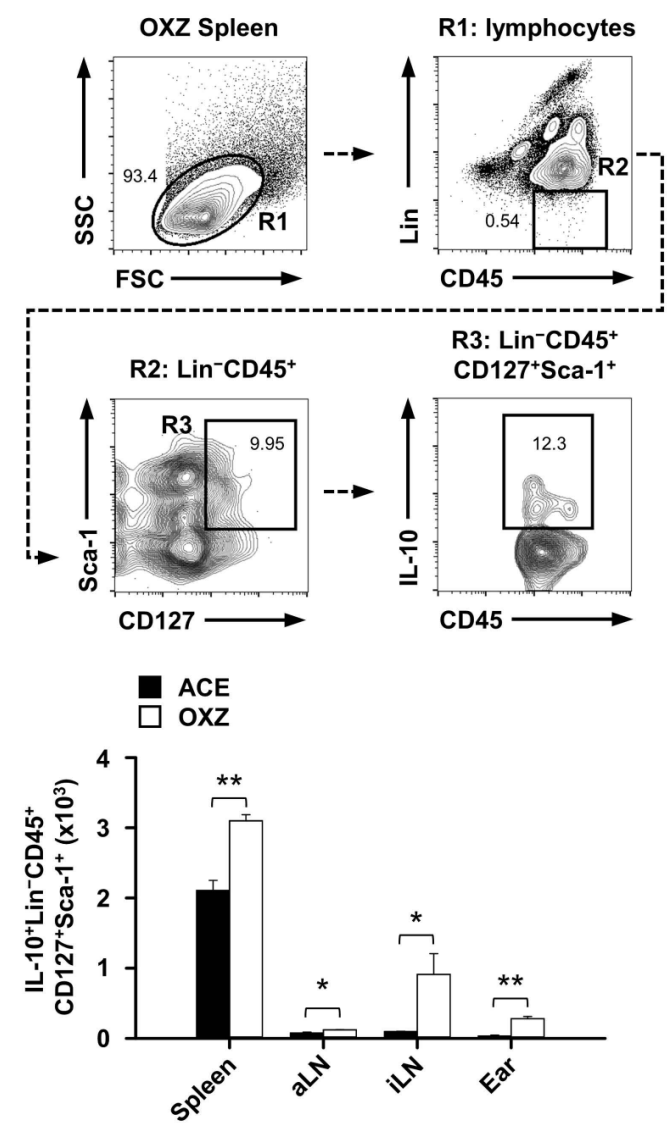

Fig. 4. Population change of IL-10-producing innate lymphoid cells in contact hypersensitivity mouse model. (A) Dot plots show a representative analysis of IL-10 expression in splenic ILCs $\left(\right.$ Lin ${ }^{-} \mathrm{CD} 45^{+} \mathrm{CD} 127^{+} \mathrm{Sca}-1^{+}$) from contact hypersensitivity mice. (B) The number of IL-10 ${ }^{+}$cells in ILCs in spleen, aLN, iLN, and ear tissues were measured by flow cytometry as described in "Materials and Methods." Data are the mean \pm SEM from three independent experiments. ${ }^{*} \mathrm{P}<0.05$; ${ }^{*} \mathrm{P}<0.01$. OXZ, ozaxolone; ACE, acetone; aLN, axillary lymph node; iLN, inguinal lymph node.

\section{Flow cytometric analysis}

Single-cell suspensions were isolated from the spleen, axillary, inguinal lymph node, and ear tissues. To detect intracellular IL-10 in lineage negative ( $\mathrm{Lin}^{-}$) or $\mathrm{Lin}^{-} \mathrm{CD} 45^{+} \mathrm{CD} 127^{+} \mathrm{Sca}-1^{+}$ cells from each tissue type, isolated cells were re-suspended and incubated for $4 \mathrm{~h}$ with Phorbol 12-Myristate 13-Acetate (PMA, $50 \mathrm{ng} / \mathrm{ml}$; Sigma), Ionomycin (500 ng/ml; Sigma), and Brefeldin A (3 mg/ml; eBioscience, San Diego, CA, USA). Before cell surface markers were stained, $\mathrm{Fc} \gamma$ receptors were blocked with Anti-CD16 and Anti-CD32 monoclonal antibodies (2.4G2, BD Biosciences). The antibodies against cell surface proteins were as follows: mouse hematopoietic lineage (anti-CD3 (17A2), anti-CD45R (B220, RA3-6B2), anti-CD11b (M1/70), anti-TER-119 (TER-119), and anti-Ly-G6 (Gr-1, RB6-8C5)), anti-CD127 (IL-7R $\alpha$, A7R34), Sca-1 (Ly-6A/E, 
D7), and anti-CD25 (PC61.5), which were purchased from eBioscience, and anti-CD45 (30-F11), which was purchased from BD Biosciences. Cells were fixed and permeabilized with a Cytofix/Cytoperm kit (eBioscience) and then were incubated with anti-IL-10 monoclonal antibody (JES5-16E3, eBioscience) at $4^{\circ} \mathrm{C}$ for $30 \mathrm{~min}$. Cells were analyzed with FACSAria or FACSCalibur flow cytometer (Becton Dickinson, San Jose, CA, USA) and Flowjo Version 10 software (TreeStar).

\section{Statistical analysis}

Data are expressed as the mean \pm SEM from at least three independent experiments. Statistical analysis was performed using one-way ANOVA and the Student's t-test for unpaired values unless stated otherwise. Statistical significance $(* \mathrm{P}<$ 0.05 and $* * P<0.01)$ was performed using the software SigmaStat (Systat Software, Inc., San Jose, CA, USA).

\section{ACKNOWLEDGEMENTS}

This work was supported by a National Research Foundation of Korea (NRF) grant funded by the Korean government (MSIP, NRF-2013-R1A4A1069575), and in part by a National Research Foundation of Korea (MSIP, NRF-2012R1A2A1A036 70516) grant (and NRF-2013R1A1A2058984).

\section{REFERENCES}

1. Koyasu S and Moro K (2012) Role of innate lymphocytes in infection and inflammation. Front Immunol 3, 101

2. Spits $\mathrm{H}$ and Cupedo $\mathrm{T}$ (2012) Innate lymphoid cells: emerging insights in development, lineage relationships, and function. Annu Rev Immunol 30, 647-675

3. Diefenbach A, Colonna M, and Koyasu S (2014) Development, differentiation, and diversity of innate lymphoid cells. Immunity 41, 354-365

4. Spits H, Artis D, Colonna M et al (2013) Innate lymphoid cells-a proposal for uniform nomenclature. Nat Rev Immunol 13, 145-149

5. Fuchs A, Vermi W, Lee JS et al (2013) Intraepithelial type 1 innate lymphoid cells are a unique subset of IL-12- and IL-15-responsive IFN- $\gamma$-producing cells. Immunity 38, 769-781

6. Neill DR, Wong SH, Bellosi A et al (2010) Nuocytes represent a new innate effector leukocyte that mediates type-2 immunity. Nature 464, 1367-1370

7. Klein Wolterink RG, Serafini N, van Nimwegen $M$ et al (2013) Essential, dose-dependent role for the transcription factor Gata3 in the development of IL-5 + and IL-13+ type 2 innate lymphoid cells. Proc Natl Acad Sci U S A 110, 10240-10245

8. Yagi R, Zhong C, Northrup DL et al (2014) The transcription factor GATA3 is critical for the development of all IL-7R $\alpha$-expressing innate lymphoid cells. Immunity 40, 378-388

9. Cupedo T, Crellin NK, Papazian N et al (2009) Human fetal lymphoid tissue-inducer cells are interleukin 17-producing precursors to RORC+ CD127+ natural killer-like cells.
Nat Immunol 10, 66-74

10. Couper KN, Blount DG and Riley EM (2008) IL-10: the master regulator of immunity to infection. J Immunol 180, 5771-5777

11. Moore KW, de Waal Malefyt R, Coffman RL and $\mathrm{O}^{\prime}$ Garra A (2001) Interleukin-10 and the interleukin-10 receptor. Annu Rev Immunol 19, 683-765

12. Saraiva $M$ and $O^{\prime}$ Garra $A$ (2010) The regulation of IL-10 production by immune cells. Nat Rev Immunol 10, 170-181

13. O'Garra A, Vieira PL, Vieira P and Goldfeld AE (2004) IL-10-producing and naturally occurring CD4+ Tregs: limiting collateral damage. J Clin Invest 114, 1372-1378

14. Chaudhry A, Samstein RM, Treuting P et al (2011) Interleukin-10 signaling in regulatory T cells is required for suppression of Th17 cell-mediated inflammation. Immunity 34, 566-578

15. Noh J, Noh G, Lee SJ et al (2012) Tolerogenic effects of interferon-gamma with induction of allergen-specific interleukin-10-producing regulatory $\mathrm{B}$ cell $(\mathrm{Br} 1)$ changes in non-IgE-mediated food allergy. Cell Immunol 273, 140-149

16. Kim HS, Lee JH, Han HD et al (2015) Autocrine stimulation of IL-10 is critical to the enrichment of IL-10-producing $\mathrm{CD} 40$ (hi)CD5(+) regulatory $\mathrm{B}$ cells in vitro and in vivo. BMB Rep 48, 54-59

17. Lee SJ, Shin SJ, Lee SJ et al (2014) Mycobacterium abscessus MAB2560 induces maturation of dendritic cells via Toll-like receptor 4 and drives Th1 immune response. BMB Rep 47, 512-517

18. Yanaba K, Bouaziz JD, Haas KM et al (2008) A regulatory B cell subset with a unique CD1dhiCD5+ phenotype controls $\mathrm{T}$ cell-dependent inflammatory responses. Immunity 28, 639-650

19. DiLillo DJ, Matsushita T and Tedder TF (2010) B10 cells and regulatory $B$ cells balance immune responses during inflammation, autoimmunity, and cancer. Ann N Y Acad Sci 1183, 38-57

20. Kim HS, Kim AR, Kim DK et al (2015) Interleukin-10producing $\mathrm{CD} 5+\mathrm{B}$ cells inhibit mast cells during immunoglobulin E-mediated allergic responses. Sci Signal 8, ra28

21. Kim AR, Kim HS, Kim DK et al (2016) Mesenteric IL-10-producing CD5(+) regulatory B cells suppress cow's milk casein-induced allergic responses in mice. Sci Rep 6, 19685

22. Gregori S, Tomasoni D, Pacciani V et al (2010) Differentiation of type $1 \mathrm{~T}$ regulatory cells (Tr1) by tolerogenic DC-10 requires the IL-10-dependent ILT4/HLA-G pathway. Blood 116, 935-944

23. Zhang X, Majlessi L, Deriaud E et al (2009) Coactivation of Syk kinase and MyD88 adaptor protein pathways by bacteria promotes regulatory properties of neutrophils. Immunity 31, 761-771

24. Robinette ML, Fuchs A, Cortez VS et al (2015) Transcriptional programs define molecular characteristics of innate lymphoid cell classes and subsets. Nat Immunol 16, 306-317

25. Wang B, Fujisawa H, Zhuang L et al (2000) CD4 + Th1 and CD8 + type 1 cytotoxic T cells both play a crucial role in the full development of contact hypersensitivity. J Immunol $165,6783-6790$ 